\title{
Multidisciplinary therapy for metastatic primary malignant melanoma of the esophagus: A case report
}

\author{
KEN SASAKI $^{1}$, YASUTO UCHIKADO ${ }^{1}$, ITARU OMOTO ${ }^{1}$, MASAHIKO AMATATSU ${ }^{1}$, \\ KOICHI MEGUMI ${ }^{1}$, HIROSHI OKUMURA ${ }^{2}$, KOSEI MAEMURA $^{1}$ and SHOJI NATSUGOE ${ }^{1}$ \\ ${ }^{1}$ Department of Digestive Surgery, Breast and Thyroid Surgery, Graduate School of Medical and Dental Sciences, \\ Kagoshima University, Kagoshima 890-8520; ${ }^{2}$ Kagoshima Kouseiren Hospital, Kagoshima 890-0061, Japan
}

Received September 24, 2017; Accepted November 13, 2017

DOI: $10.3892 /$ mco.2018.1572

\begin{abstract}
Standard treatment strategies have not yet been established for primary malignant melanoma of the esophagus (PMME), and far much less for recurrent disease. There are no reports of anti- programmed death-1 antibody treatment of recurrent PMME. A 60-year-old Japanese man was diagnosed with a primary malignant melanoma in the lower esophagus. The patient underwent mediastinoscope-assisted subtotal esophagectomy, and two nodal involvements were detected in the lymph nodes (LN)s along the left gastric artery. Paclitaxel and oral fluoropyrimidine were administered for 2 months as adjuvant treatment based on results of a histoculture drug response assay. Computed tomography at 8 months after following surgery revealed LN metastasis around the celiac axis. The serum level of the tumor marker 5-S-cysteinyldopa was elevated aberrantly. Although treatment with dacarbazine and interferon- $\beta$ was initiated, metastatic disease progressed. Therefore, we started anti-programmed death-1 antibody therapy. Following 8 treatment courses, the patient demonstrated a partial response; however, after following 4 more treatment courses, the patient demonstrated progressive disease. Next, hypofractionated radiotherapy was targeted at the metastatic LN and resulted in a partial response. Then, ipilimumab, an anticytotoxic T-lymphocyte associated antigen 4 , was administered
\end{abstract}

Correspondence to: Dr Ken Sasaki, Department of Digestive Surgery, Breast and Thyroid Surgery, Graduate School of Medical and Dental Sciences, Kagoshima University, 8-35-1 Sakuragaoka, Kagoshima 890-8520, Japan

E-mail:k-sasaki@m.kufm.kagoshima-u.ac.jp

Abbreviations: PMME, primary malignant melanoma of the esophagus; PD-1, programmed death 1; CTLA-4, cytotoxic T-lymphocyte associated antigen 4; PD-L1: programmed death ligand 1; CT, computed tomography; PD, progressive disease; RECIST, response evaluation criteria in solid tumors; PR, partial response; RT, radiotherapy; OS, overall survival

Key words: anti-PD-1 antibody, hypofractionated radiotherapy, primary malignant melanoma of the esophagus, LN metastasis, multidisciplinary therapy at a dose of $3 \mathrm{mg} / \mathrm{kg}$. After the initial administration of ipilimumab, grade 3 peripheral neuropathy was recognized; thereafter, ipilimumab was not administered. A total of 18 months after following treatment for metastatic LNs, the LN decreased in size, and there were no other signs of metastasis to other organs. The patient then underwent laparoscopic celiac axis lymphadenectomy. Pathological examination of the surgical specimens identified no viable melanoma cells. A total of 8 months after following surgery, he is free from evidence of disease recurrence. This is the first reported case of recurrent PMME successfully treated with multidisciplinary therapy including anti-programmed death-1 antibody therapy, radiotherapy and laparoscopic lymphadenectomy.

\section{Introduction}

Primary malignant melanoma of the esophagus (PMME) accounts for only 0.1 to $0.3 \%$ of all esophageal neoplasms (1-3), and the prognosis is extremely poor because of early hematogenous and lymphatic metastases. Standard treatment strategies and clear guidelines have not been established for PMME, much less for recurrent disease. There have been major recent advances in the management of metastatic melanoma, including immune checkpoint inhibitors, such as anti-programmed death-1 (anti-PD-1) and anti-CTLA-4 antibodies. In particular, targeting the PD-1 pathway in patients with metastatic melanoma has demonstrated a substantial clinical benefit $(4,5)$. However, there are currently no reports on the use of the antiPD-1 antibody, nivolumab, followed by radiotherapy in patients with recurrent PMME. Here, we report a case of PMME that recurred in the lymph node (LN) around the celiac axis after thoracoscopic esophagectomy, which was treated with multidisciplinary therapy with dacarbazine, monoclonal antibodies directed against negative regulators such as PD-1, hypofractionated radiotherapy and laparoscopic lymphadenectomy.

\section{Case report}

A 60-year-old Japanese man who presented with a chief complaint of dysphagia to solid foods was diagnosed with PMME in the lower esophagus. Because the patient had a prior history of right upper lobectomy for lung cancer, he underwent mediastinoscope-assisted subtotal esophagectomy 
with two-field LN dissection and gastric tube reconstruction via the mediastinal route. Evaluation of the resected specimen demonstrated that the tumor invaded the muscularis propria (pT2), and two nodal involvements were detected in the LNs along the left gastric artery (pN1). The UICC pathological staging for his PMME was IIA, T2, pN1, pM0. The resected specimen showed an elevated polypoid tumor $85 \times 55 \mathrm{~mm}$ in size (Fig. 1). Histopathologically, tumor cells consisted of malignant large tumor cells with abundant eosinophilic cytoplasm, and contained few melanin granules on hematoxylin and eosin (H\&E) staining (Fig. 2A). Subsequent immunohistology revealed that the tumor cells were positive for melan-A (Fig. 2B), HMB-45 (Fig. 2C), and S-100 (Fig. 2D) and negative for cytokeratin markers, AE1/AE3 (Fig. 2E), resulting in a diagnosis of PMME. Further analysis revealed a membranous staining pattern for programmed death ligand 1(PD-L1) (Fig. 2F). The percentage of PD-L1 positive tumor cells within resected specimen was $\sim 10 \%$ (anti-PD-L1 antibody; clone 28-8; ab205921; Abcam). No BRAF mutations were detected by direct sequencing analysis. Paclitaxel and S-1, an oral dihydropyrimidine-dehydrogenaseinhibitory fluoropyrimidine, were administered for 2 months as adjuvant therapy based on the results of a histoculture drug response assay (HDRA), which is a clinically practical in vitro drug-response assay for identifying optimal anticancer agents. Eight months after surgery, computed tomography (CT) revealed a 19-mm-diameter, oval-shaped mass in the LN around the celiac axis (Fig. 3A), and ${ }^{18} \mathrm{~F}$-fluorodeoxyglucose positron-emission tomography/CT (FDG-PET/CT) showed intense FDG uptake in the lesion (Fig. 3B). No abnormal uptake was found at any other site of the body. Thus, based on imaging study findings, we diagnosed recurrence of disease in the LN around the celiac axis. The level of 5-S-CD was $12.6 \mathrm{nmol} / 1$ (reference value, $1.5-8 \mathrm{nmol} / \mathrm{l}$ ) at the time of diagnosis of recurrent disease. Although there was only one site of recurrence, we first initiated nonoperative management because of a high rate of relapse. The patient received the first treatment session with dacarbazine $\left(1,000 \mathrm{mg} / \mathrm{m}^{2}\right.$, day 1$)$ and interferon $\beta$ (300 units/day, days 1-10); no substantial adverse effects were observed. CT performed after 4 courses after chemotherapy revealed progressive disease (PD) of the metastatic LN lesion according to response evaluation criteria in solid tumors (RECIST) (Fig. 3C). Second, nivolumab, an anti-PD-1 antibody, was administered at a dose of $2 \mathrm{mg} / \mathrm{kg}$ every 3 weeks. After 8 treatment courses, CT revealed a partial response (PR) of the LN lesion (Fig. 3D); however, after 4 more treatment courses, CT revealed PD of the LN lesion. During the first courses of nivolumab treatment, hyperthyroidism was observed, and predonizoron and potassium iodide were used to treat hyperthyroidism. Third, hypofractionated radiotherapy (RT) (4,000 cGy divided in 8 fractions) was targeted at the metastatic LN and resulted in a PR (Fig. 3E and F); no substantial adverse effects were observed. Fourth, ipilimumab, an anti-CTLA-4 antibody, was given at a dose of $3 \mathrm{mg} / \mathrm{kg}$. After initial administration of ipilimumab, grade 3 peripheral neuropathy [defined by National Cancer Institute Common Terminology Criteria for Adverse Events (NCI-CTCAE), version 4.0] was recognized; thereafter, ipilimumab was not administered. Eighteen months after treatment for the metastatic LN, the LN decreased in size, and there were no other signs of metastasis to other organs. The patient then underwent laparoscopic celiac axis lymphadenectomy, and had no post-operative complications. Pathologic examination of the surgical specimens identified no viable melanoma cells (Fig. 4A and B). Eight months after surgery, he is free from evidence of local and distant disease recurrence (Fig. 4C). shows the clinical course and changes in the tumor marker 5-S-CD and in tumor size is shown in Fig. 5. Written informed consent was obtained from the patient.

\section{Discussion}

Metastatic melanoma is particularly difficult to cure because it shows resistance to therapies. An abdominal LN metastasis was detected 8 months after surgery, although the present case received adjuvant therapy for the prevention of cancer recurrence. Despite recent advances in melanoma treatment, interferon alpha is the only therapy currently licensed for the adjuvant treatment of melanoma, with documented success in improving recurrence-free survival and, to a lesser extent, overall survival (OS) (6). Therefore, adjuvant treatment based on the results of HDRA, which was developed as an in vitro drug-response assay for choosing anticancer agents (7), was given. The tumor inhibition rates of chemotherapy agents evaluated by the HDRA were found to be predictive of the response of various types of cancer to chemotherapy (8-10). Conventionally, a cytotoxic agent such as dacarbazine has been used for metastatic malignant melanoma. However, it is hard to say that dacarbazine has a high response rate. Inhibition of the PD-1 pathway by nivolumab improves OS compared with dacarbazine in advanced melanoma (5). Primary anorectal (11) and lung (12) malignant melanomas successfully treated with nivolumab were reported; however, to the best of our knowledge, this is the first case report regarding the administration of anti-PD-1 antibody followed by definitive hypofractionated RT for recurrent PMME. We administered dacarbazine as first-line therapy; however, we considered that dacarbazine therapy was not effective, and we started nivolumab as a second-line therapy. Nivolumab markedly reduced the tumor size. In this case, PD-L1 expression in tumor cells supported a response to nivolumab. Although malignant melanoma is generally considered to be radioresistant, hypofractionated RT led to a favorable outcome. Preclinical evidence suggests that PD-1 blockade may interact with RT to improve local tumor control in melanoma (13) and survival in glioma (14) in a variety of radiation dose and fractionation schema in breast cancer (15). Furthermore, Park et al reported that PD-1 blockade or deficiency can synergize with local radiotherapy to induce tumor-specific CD8-positive T-cell immunity (16). Only 3 clinical studies reported to date have focused on the relationship between anti-PD-1 antibody and RT. A retrospective cohort study reported that control of distant brain metastases and OS may be improved with antiPD-1 antibody therapy and stereotactic RT compared with RT and/or surgery alone. Control of distant brain metastases and OS were not affected by the timing of anti-PD-1 antibody administration before, during or after stereotactic RT (17). A case series reported that neoadjuvant treatment for stage III/IV melanoma with anti-PD-1 antibody and hypofractionated RT had substantial clinical benefit without significant toxicity (18). 


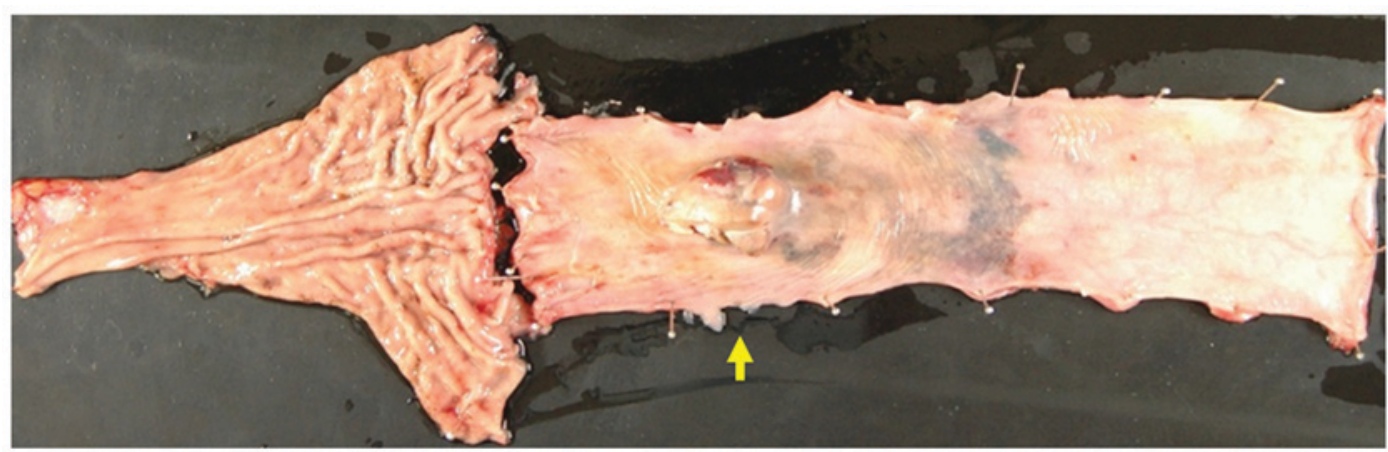

Figure 1. Macroscopic view of the excised specimen shows an elevated polypoid tumor 85 x $55 \mathrm{~mm}$ in size.
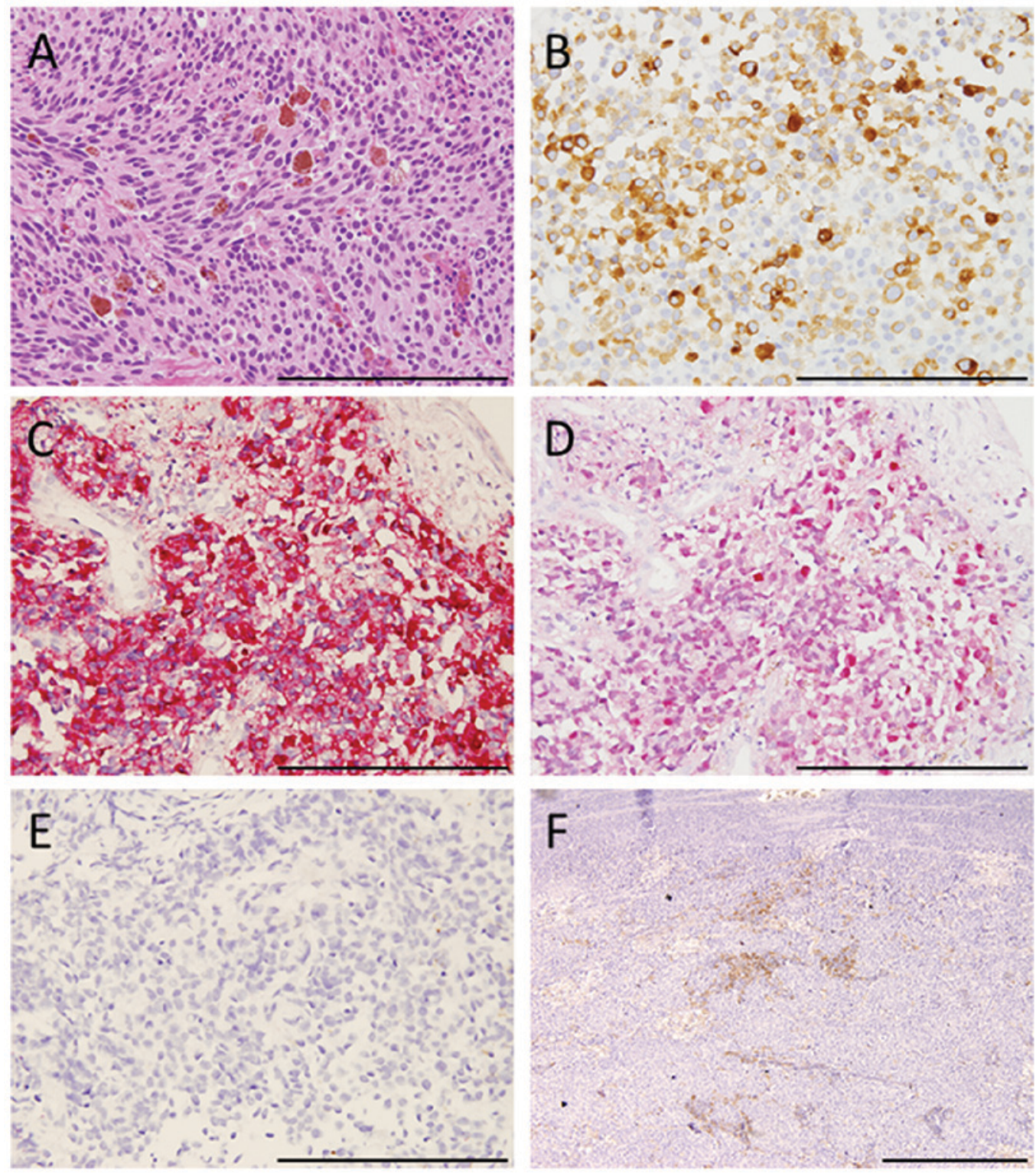

Figure 2. Histopathological findings. (A) Tumor cells stained with hematoxylin and eosin. (B) Immunohistochemical staining of tumor cells was positive for melan-A, (C) HMB-45, and (D) S-100 and negative for cytokeratin markers, (E) AE1/AE3 [(A-E) original magnification, x400. Scale bar, 200 $\mu \mathrm{m}$ ]. (F) Immunohistochemical membranous positive staining of PD-L1 in tumor cells (original magnification, x100; scale bar, $500 \mu \mathrm{m}$.

A case report showed an abscopal effect, a rare phenomenon of tumor regression at a site distant from the primary site of radiotherapy (19), when radiotherapy was added to ongoing
anti-CTLA-4 antibody therapy in a patient with melanoma (20). Prospective clinical studies of RT and anti-PD-1 antibody therapy in patients with melanoma and other malignancies are 

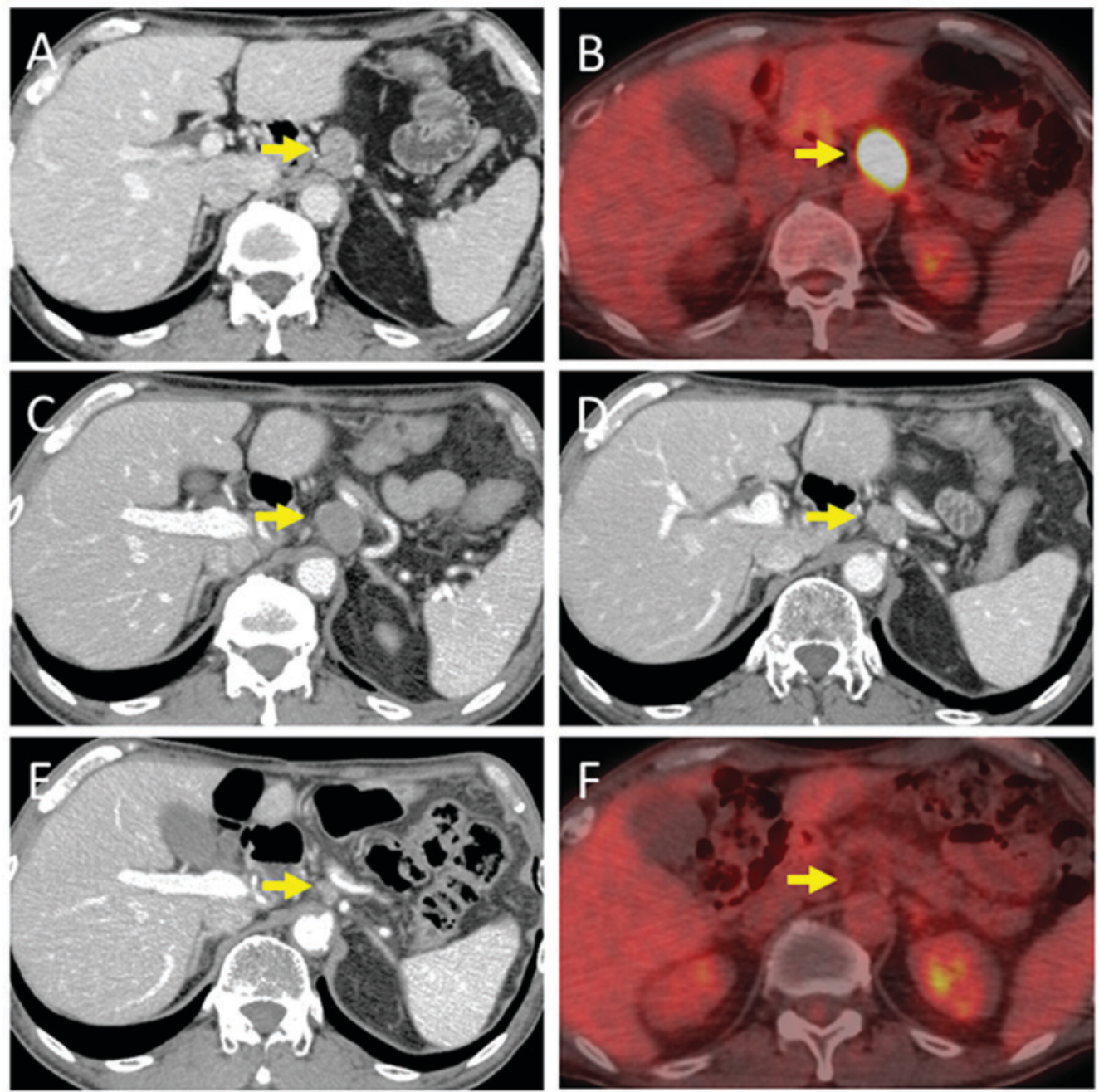

Figure 3. Computed tomography (CT) and ${ }^{18} \mathrm{~F}$-fluorodeoxyglucose positron-emission tomography/computed tomography (FDG-PET/CT). (A) Pre-treatment CT showing the metastatic lymph node (LN) around the celiac axis. (B) Pre-treatment FDG-PET/CT showing abnormal uptake of FDG in the metastatic LN $\left(\mathrm{SUV}_{\max }, 26.8\right)$. (C) CT showing the bigger metastatic LN after 4 courses of chemotherapy with dacarbazine and interferon $\beta$. (D) CT showing the smaller metastatic LN after 12 courses of nivolumab therapy. (E) CT showing the smaller metastatic LN after radiotherapy. (F) FDG-PET/CT showing no uptake of FDG in the LN after radiotherapy.
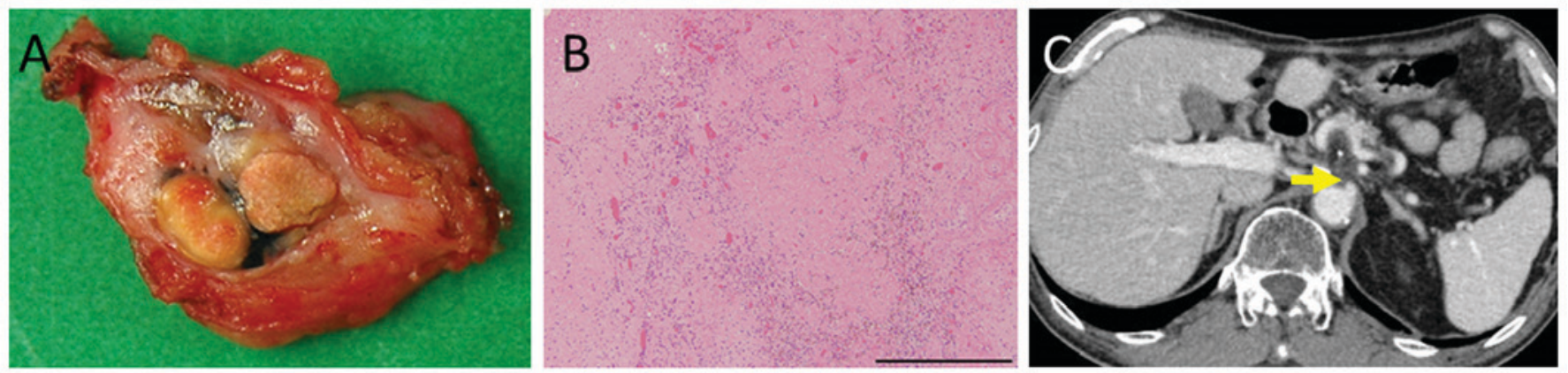

Figure 4. Pathological findings of the surgical supecimen and CT image after celiac axis lymphadenectomy. (A) Macroscopic view of the resected lymph nodes. (B) Resected lymph nodes stained with hematoxylin and eosin. Necrosis and hyalinosis were observed in resected lymph nodes [(B) original magnification, x100. Scale bar, $500 \mu \mathrm{m}$.]. (C) CT showing the no LN recurrence after lymphadenectomy.

warranted. Anti-CTLA4 antibody therapy was additionally performed, although anti-PD-1 antibody therapy and RT have resulted in a PR. PMME is an extremely difficult malignancy because of early hematogenous and lymphatic metastases. Therefore, we did not intend to perform lymphadenectomy at first, and performed anti-CTLA4 antibody therapy to obtain complete response. However, severe peripheral neuropathy was recognized at the initial dose, and we determined to perform lymphadenectomy. The limitation of this case study is that we have not been able to show if anti-PD1 antibody was effective against PMME. Total remission was achieved rather after radiotherapy, however, we think that not only 


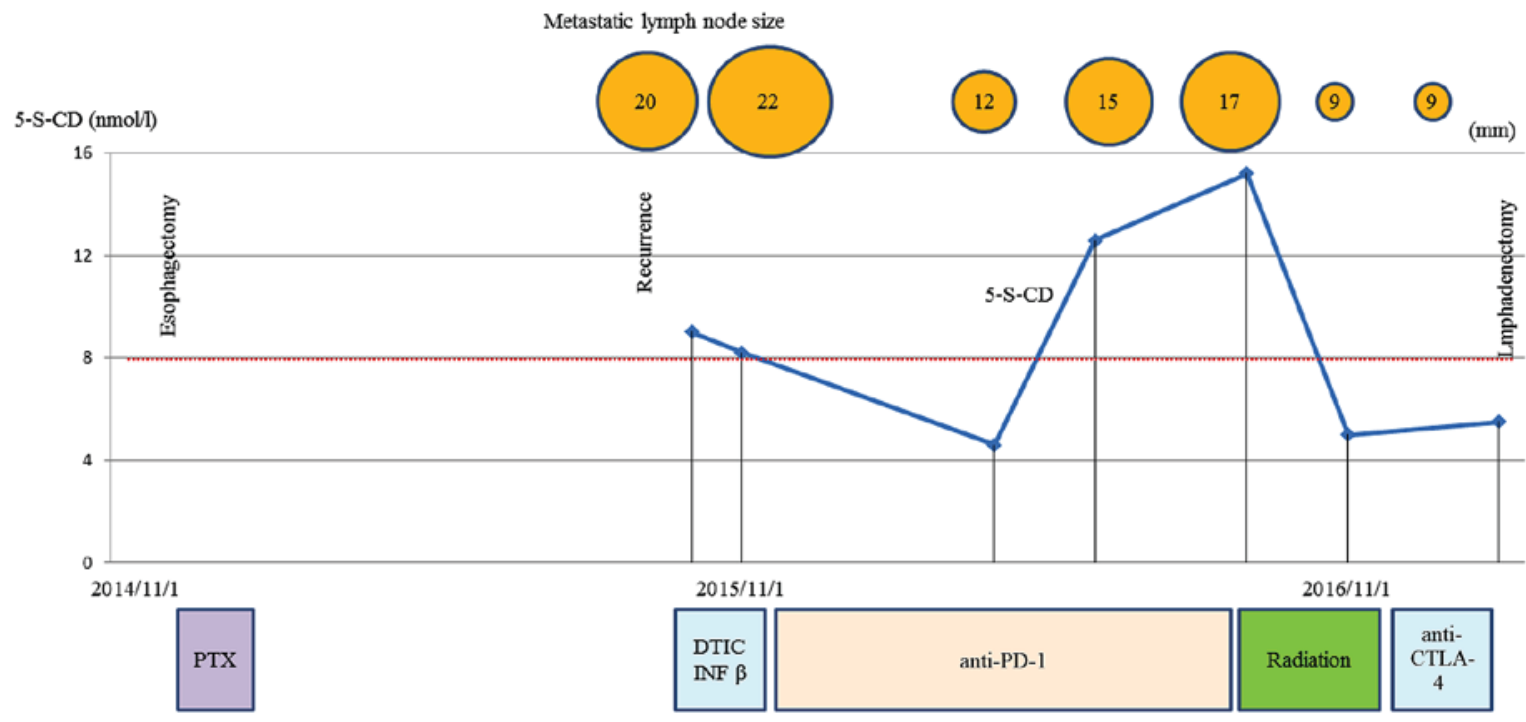

Figure 5. Clinical course and changes in the tumor marker 5-S-CD and tumor size. PTX, paclitaxel; DTIC, dacarbazine; INF $\beta$, interferon $\beta$.

radiotherapy but also multidisciplinary therapy including anti-PD-1 antibody therapy were effective for PMME in this case because malignant melanoma is generally considered to be radioresistant, and there are some reports about synergic effect of RT and anti-PD-1 antibody (13-18).

In conclusion, we report the first case of recurrent PMME to be treated with combinations of chemotherapy, immunotherapy, RT and laparoscopic lymphadenectomy. This finding indicates that the combination of cytotoxic and moleculartargeted chemotherapy and RT may be suitable for select patients with metastatic PMME. Additional studies are needed to establish the usefulness of anti-PD-1antibody therapy for metastatic PMME.

\section{References}

1. Makuuchi H, Takubo K, Yanagisawa A and Yamamoto S: Esophageal malignant melanoma: Analysis of 134 cases collected by the Japan Esophageal Society. Esophagus 12: 158-169, 2015.

2. Terada T: A clinicopathologic study of esophageal 860 benign and malignant lesions in 910 cases of consecutive esophageal biopsies. Int J Clin Exp Pathol 6: 191-198, 2013.

3. Sabanathan S, Eng J and Pradhan GN: Primary malignant melanoma of the esophagus. Am J Gastroenterol 84: 1475-1481, 1989.

4. Weber JS, D’Angelo SP, Minor D, Hodi FS, Gutzmer R, Neyns B, Hoeller C, Khushalani NI, Miller WH Jr, Lao CD, et al: Nivolumab versus chemotherapy in patients with advanced melanoma who progressed after anti-CTLA-4 treatment (CheckMate 037): A randomised, controlled, open-label, phase 3 trial. Lancet Oncol 16: 375-384, 2015.

5. Robert C, Long GV, Brady B, Dutriaux C, Maio M, Mortier L, Hassel JC, Rutkowski P, McNeil C, Kalinka-Warzocha E, et al: Nivolumab in previously untreated melanoma without BRAF mutation. N Engl J Med 372: 320-330, 2015.

6. Mocellin S, Pasquali S, Rossi CR and Nitti D: Interferon alpha adjuvant therapy in patients with high-risk melanoma: A systematic review and meta-analysis. J Natl Cancer Inst 102: 493-501, 2010.

7. Furukawa T, Kubota T, Watanabe M, Takahara T, Yamaguchi H, Takeuchi T, Kase S, Kodaira S, Ishibiki K, Kitajima M, et al: High in vitro-in vivo correlation of drug response using spongegel-supported three-dimensional histoculture and the MTT end point. Int J Cancer 51: 489-498, 1992.

8. Furukawa T, Kubota T and Hoffman RM: Clinical applications of the histoculture drug response assay. Clin Cancer Res 1: 305-311, 1995 .
9. Kubota T, Sasano N, Abe O, et al: Potential of the histoculture drug-response assay to contribute to cancer patient survival. Clin Cancer Res 1: 1537-1543, 1995

10. Fujita Y, Hiramatsu M, Kawai M, Nishimura H, Miyamoto A and Tanigawa N: Histoculture drug response assay predicts the postoperative prognosis of patients with esophageal cancer. Oncol Rep 21: 499-505, 2009.

11. Tokuhara K, Nakatani K, Tanimura H, Yoshioka K, Kiyohara T and Kon M: A first reported case of metastatic anorectal amelanotic melanoma with a marked response to anti-PD-1 antibody nivolumab: A case report. Int J Surg Case Rep 31: 188-192, 2017.

12. Hirai I, Tanese K, Obata S and Funakoshi T: A case of primary malignant melanoma of the lung responded to anti-PD-1 antibody therapy. Indian J Thorac Cardiovasc Surg 33: 1-3, 2017.

13. Liang H, Deng L, Chmura S, Burnette B, Liadis N, Darga T, Beckett MA, Lingen MW, Witt M, Weichselbaum RR, et al: Radiation-induced equilibrium is a balance between tumor cell proliferation and T cell-mediated killing. J Immunol 190: 5874-5881, 2013.

14. Zeng J, See AP, Phallen J, et al: Anti-PD-1 blockade and stereotactic radiation produce long-term survival in mice with intracranial gliomas. International Journal of Radiation Oncol Biol Phys 86: 343-349, 2013.

15. Verbrugge I, Hagekyriakou J, Sharp LL, Galli M, West A, McLaughlin NM, Duret H, Yagita H, Johnstone RW, Smyth MJ, et al: Radiotherapy increases the permissiveness of established mammary tumors to rejection by immunomodulatory antibodies. Cancer Res 72: 3163-3174, 2012.

16. Park SS, Dong H, Liu X, Harrington SM, Krco CJ, Grams MP, Mansfield AS, Furutani KM, Olivier KR and Kwon ED: PD-1 restrains radiotherapy-induced abscopal effect. Cancer Immunol Res 3: 610-619, 2015.

17. Ahmed KA, Stallworth DG, Kim Y, Johnstone PAS, Harrison LB, Caudell JJ, Yu HHM, Etame AB, Weber JS and Gibney GT: Clinical outcomes of melanoma brain metastases treated with stereotactic radiation and anti-PD-1 therapy. Ann Oncol 27: 434-441, 2016

18. Alevizakos M, Ollila DW, Chera BS, Dodd LG, Kish JB and Moschos SJ: Combined modality neoadjuvant treatment for stage III/IV melanoma with PD-1 blockade plus radiation: A case series. Cancer Treat Res Commun 10: 12-16, 2017.

19. Mole R: Whole body irradiation-Radiobiology or medicine? Br J Radiol 26: 234-241, 1953.

20. Postow MA, Callahan MK, Barker CA, Yamada Y, Yuan J, Kitano S, Mu Z, Rasalan T, Adamow M, Ritter E, et al: Immunologic correlates of the abscopal effect in a patient with melanoma. N Engl J Med 366: 925-931, 2012. 